\title{
On Bipolar Anti Q- Fuzzy Groups
}

\author{
${ }^{1}$ A.Solairaju \\ ${ }^{2}$ R.Nagarajan \\ ${ }^{3}$ P.Muruganantham \\ ${ }^{4}$ M.Geethalakshmi \\ 1. PG \& Research Department of Mathematics, Jamal Mohamed College, Trichy-20 \\ 2. Department of Mathematics, JJ College Of Engg \& Tech, Trichy- 09 \\ 3. Department of Mathematics, Kurinji College of Arts \& Science, Trichy-02 \\ 4. Department of Mathematics, Jayaram College of Engg \& Tech, Thuraiyur.
}

\begin{abstract}
In this paper, we apply the notion of bipolar-valued Q- fuzzy set to groups. We introduce the concept of bipolar anti Qfuzzy groups / anti Q-fuzzy d-ideals of groups and investigate several properties. We give relations between a bipolar anti Q-fuzzy group and bipolar anti Q-fuzzy d-ideal. We provide a condition for a bipolar anti Q- fuzzy groups to be a bipolar anti Q- fuzzy d-ideal. We also give characterizations of bipolar anti Q- fuzzy ideal. We consider the concept of strongest bipolar anti Q- fuzzy relations on bipolar anti Q-fuzzy d-ideals of a group and discuss some related properties.
\end{abstract}

AMS Subject Classification (2000): 06F35, 03G25, 08A72, $20 \mathrm{~N} 25$

Index terms: Bipolar fuzzy group, Bi polar anti Q- fuzzy group, bipolar anti fuzzy d-ideal, (t, s)- bipolar strongest fuzzy relation.

\section{INTRODUCTION}

Fuzzy sets are a kind of useful mathematical structure to represent a collection of objects whose boundary is vague. There are several kinds of fuzzy sets extensions in the fuzzy set theory, for example, intuitionistic fuzzy sets, interval valued fuzzy sets, vague sets etc. Bipolar-valued fuzzy sets are an extension of fuzzy sets whose membership degree range is enlarged from the interval $[0,1]$ to $[-1,1]$. Bipolar-Valued fuzzy sets have membership degrees that represent the degree of satisfaction to the property and its counter property. In a bipolar valued fuzzy set the membership degree 0 means that elements are irrelevant to the corresponding property, the membership degrees on $(0,1]$ indicate that elements some what satisfy the property, and the membership degrees on $[-1,0)$ indicate that elements somewhat satisfy the implicit counter property [sec(3)]. In the definition of bipolar-valued fuzzy sets, there are two kinds of representations so called canonical representation and reduced representation. In this paper, we use the canonical representation of bipolar valued Q- fuzzy sets. In this paper, In this paper, we apply the notion of bipolar-valued Q- fuzzy set to groups. We introduce the concept of bipolar anti Qfuzzy groups / anti Q-fuzzy d-ideals of groups and investigate several properties. We give relations between a bipolar anti Q-fuzzy group and bipolar anti Q-fuzzy d-ideal. We provide a condition for a bipolar anti Q- fuzzy groups to be a bipolar anti Q- fuzzy d-ideal. We also give characterizations of bipolar anti Q- fuzzy ideal. We consider the concept of strongest bipolar anti Q- fuzzy relations on bipolar anti Q-fuzzy d-ideals of a group and discuss some related properties.

\section{PRELIMINARIES}

2.1 Definition: Let ' $S$ ' be a set. A Q- fuzzy set in $S$ is a function $\mu: \mathrm{S} \times \mathrm{Q} \rightarrow[0,1]$

2.2 Definition: Let ' $G$ ' be a non-empty set. A bipolar-Valued Q-Fuzzy set $\mathrm{A}$ in $\mathrm{G}$ is an object having the form;

$\mathrm{A}=\left\{\left((\mathrm{x}, \mathrm{q}) \mu_{\mathrm{A}}^{+}(\mathrm{x}, \mathrm{q}), \mu_{\mathrm{A}}^{-}(\mathrm{x}, \mathrm{q})\right) / \mathrm{x} \varepsilon \mathrm{G}, \mathrm{q} \varepsilon \mathrm{Q}\right\}$ where $\mu_{\mathrm{A}}{ }^{+}: \mathrm{G} \times \mathrm{Q} \rightarrow[0,1]$ and $\mu_{\mathrm{A}}{ }^{-}: \mathrm{G} \times \mathrm{Q} \rightarrow[-1,0]$ are mapping. The positive membership degree $\mu_{\mathrm{A}}^{+}(\mathrm{x}, \mathrm{q})$ denotes the satisfaction degree of an element $\mathrm{x}$ to the property corresponding to ' $\mathrm{A}$ ' and the negative membership degree $\mu_{\mathrm{A}}$ ' $(\mathrm{x}, \mathrm{q})$ denotes the satisfaction degree of $\mathrm{x}$ to some implicit counter property of $\mathrm{A}$.

2.3 Definition: A bipolar Q- fuzzy set (BFS) ' $A$ ' in $X$ is called a bipolar Q- Fuzzy group (BGFG) of $\mathrm{X}$ if it satisfies

$\left(\mathrm{BQFG}_{1}\right) \mu_{\mathrm{A}}^{+}(\mathrm{xy}, \mathrm{q}) \geq \min \left\{\mu_{\mathrm{A}}^{+}(\mathrm{x}, \mathrm{q}), \mu_{\mathrm{A}}^{+}(\mathrm{y}, \mathrm{q})\right\}$ $\left(\mathrm{BQFG}_{2}\right) \mu_{\mathrm{A}}^{-}(\mathrm{xy}, \mathrm{q}) \leq \max \left\{\mu_{\mathrm{A}}^{-}(\mathrm{x}, \mathrm{q}), \mu_{\mathrm{A}}^{-}(\mathrm{y}, \mathrm{q})\right\}$ 
$\varepsilon Q$

$\left(\mathrm{BQFG}_{3}\right) \mu_{\mathrm{A}}^{+}\left(\mathrm{x}^{-1}, \mathrm{q}\right) \geq \mu_{\mathrm{A}}^{+}(\mathrm{x}, \mathrm{q})$ and $\left(\mathrm{BQFG}_{4}\right) \mu_{\mathrm{A}}^{-}\left(\mathrm{x}^{-1}, \mathrm{q}\right) \leq \mu_{\mathrm{A}}^{-}(\mathrm{x}, \mathrm{q})$ for all $\mathrm{x}, \mathrm{y} \varepsilon \mathrm{X}$ and $\mathrm{q}$

2.4 Definition: A bipolar fuzzy set ' $A$ ' in $X$ is called a bipolar anti Q- Fuzzy group (BAGFG) of $\mathrm{X}$ if it satisfies

$$
\begin{aligned}
& \left(\mathrm{BAQFG}_{1}\right) \mu_{\mathrm{A}}^{+}(\mathrm{xy}, \mathrm{q}) \leq \mathrm{T}\left\{\mu_{\mathrm{A}}^{+}(\mathrm{x}, \mathrm{q}), \mu_{\mathrm{A}}^{+}(\mathrm{y}, \mathrm{q})\right\} \\
& \left(\mathrm{BAQFG}_{2}\right) \mu_{\mathrm{A}}^{-}(\mathrm{xy}, \mathrm{q}) \geq \mathrm{S}\left\{\mu_{\mathrm{A}}^{-}(\mathrm{x}, \mathrm{q}), \mu_{\mathrm{A}}^{-}(\mathrm{y}, \mathrm{q})\right\} \\
& \left(\mathrm{BAQFG}_{3}\right) \mu_{\mathrm{A}}^{+}\left(\mathrm{x}^{-1}, \mathrm{q}\right) \leq \mu_{\mathrm{A}}^{+}(\mathrm{x}, \mathrm{q}) \text { and } \\
& \left(\mathrm{BAQFG}_{4}\right) \mu_{\mathrm{A}}^{-}\left(\mathrm{x}^{-1}, \mathrm{q}\right) \geq \mu_{\mathrm{A}}{ }^{-}(\mathrm{x}, \mathrm{q}) \text { for all } \mathrm{x}, \mathrm{y} \varepsilon \mathrm{X} . \mathrm{q} \varepsilon
\end{aligned}
$$

Q

2.5 Definition: For a bipolar Q- fuzzy set 'A' and $(\beta, \alpha) \varepsilon[-$ $1.0] \times[0,1]$, we define

$$
\begin{aligned}
\mathrm{A}_{\mathrm{t}}^{+}=\left\{\mathrm{x} \varepsilon \mathrm{X} / \mu_{\mathrm{A}}^{+}(\mathrm{x}, \mathrm{q}) \leq \alpha\right\} \\
\mathrm{A}_{\mathrm{S}}^{-}=\left\{\mathrm{x} \varepsilon \mathrm{X} / \mu_{\mathrm{A}}^{-}(\mathrm{x}, \mathrm{q}) \geq \alpha\right\}
\end{aligned}
$$

which are called the positive t-cut and negative s-cut of $\mathrm{A}$ respectively.

2.6 Definition: A bipolar Q- fuzzy set ' $A$ ' in $\mathrm{X}$ is called a bipolar anti Q- fuzzy d-ideal (BAGFI) of $X$ if it satisfies;

$$
\begin{aligned}
& \left(\mathrm{BQAFI}_{1}\right) \mu_{\mathrm{A}}^{+}(\mathrm{x}, \mathrm{q}) \leq \mathrm{T}\left\{\mu_{\mathrm{A}}^{+}(\mathrm{xy}, \mathrm{q}), \mu_{\mathrm{A}}^{+}(\mathrm{y}, \mathrm{q})\right\} \\
& \left(\mathrm{BQAFI}_{2}\right) \mu_{\mathrm{A}}^{-}(\mathrm{x}, \mathrm{q}) \geq \mathrm{S}\left\{\mu_{\mathrm{A}}^{-}(\mathrm{xy}, \mathrm{q}), \mu_{\mathrm{A}}^{-}(\mathrm{y}, \mathrm{q})\right\} \\
& \left(\mathrm{BQAFI}_{3}\right) \mu_{\mathrm{A}}^{+}(\mathrm{e}, \mathrm{q}) \leq \mu_{\mathrm{A}}^{+}(\mathrm{x}, \mathrm{q}) \text { and } \mu_{\mathrm{A}}^{-}(\mathrm{e}, \mathrm{q}) \geq \mu_{\mathrm{A}}^{-}
\end{aligned}
$$

$$
(\mathrm{x}, \mathrm{q}) \text { and for all } \quad \mathrm{x}, \mathrm{y} \varepsilon \mathrm{X} \text { and } \mathrm{q} \varepsilon \mathrm{Q} \text {. }
$$

2.7 Definition: Let $\lambda$ and $\mu$ be two Q-fuzzy subsets in $X$. The Cartesian Product of $\lambda^{+} \times \mu^{+}: \mathrm{X} \times \mathrm{X} \rightarrow[0,1]$ is defined by $\lambda^{+} \times \mu^{+}(\mathrm{x}, \mathrm{y})_{\mathrm{q}}=\mathrm{T}\left\{\lambda^{+}(\mathrm{x}, \mathrm{q}), \mu^{+}(\mathrm{y}, \mathrm{q})\right\}$ and

$\lambda^{+} \times \mu^{+}: X \times X \rightarrow[0,1]$ is defined by $\lambda^{+} \times \mu^{+}(\mathrm{x}, \mathrm{y})_{\mathrm{q}}=\mathrm{S}\left\{\lambda^{-}\right.$ $(\mathrm{x}, \mathrm{q}), \mu(\mathrm{y}, \mathrm{q})\}$ for all

$$
\mathrm{x}, \mathrm{y} \varepsilon \mathrm{X} \text { and } \mathrm{q} \varepsilon \mathrm{Q} \text {. }
$$

2.8 Definition: Let $\mathrm{f}: \mathrm{X} \rightarrow \mathrm{Y}$ be a mapping of group's and ' $\mu$ ' be a bipolar Q- fuzzy set of Y. The map $\mu^{\mathrm{f}}$ is the pre image of $\mu_{1}$ and $\mu_{2}$ under f. If $\mu_{1}^{+\mathrm{f}}(\mathrm{x}, \mathrm{q})=\mu^{+\mathrm{f}}(\mathrm{x}, \mathrm{q})$, $\mu_{2}^{-\mathrm{f}}(\mathrm{x}, \mathrm{q})=\mu^{-\mathrm{f}}(\mathrm{x}, \mathrm{q})$.

2.9 Definition: Let ' $A$ ' be a bipolar Q- fuzzy set in a $X$, the strongest bipolar Q- fuzzy relation on $\mathrm{X}$ that is fuzzy relation on $\mathrm{A}$ is $\mu_{\mathrm{A}}$ given by,

$$
\begin{aligned}
& \mu_{A}^{+}(x, y)_{q}=T\left\{A^{+}(x, q), A^{+}(y, q)\right\} \\
& \mu_{A}^{-}(x, y)_{q}=S\left\{A^{-}(x, q), A^{-}(y, q)\right\} \text { for all } x, y \varepsilon X \text { and } q
\end{aligned}
$$

$\varepsilon \mathrm{Q}$.

For the sake of simplicity, we shall use the symbol $A=\left(\mu^{+}, \mu\right.$ ) for the bipolar valued Q-fuzzy set $A=\left\{\left((x, q), \mu^{+}(x, q), \mu^{-}\right.\right.$ $(\mathrm{x}, \mathrm{q})) / \mathrm{x} \varepsilon \mathrm{G})$ and (BQFRG) for bipolar Q- fuzzy regular group of $\mathrm{G}$.

\section{PROPERTIES OF BIPOLAR ANTI Q- FUZZY GROUP AND BIPOLAR ANTI Q- FUZZY D-IDEALS}

Proposition 3.1: If $\phi$ is a bipolar Anti Q- fuzzy group of $X$, then $\mu_{\varphi}^{+}(\mathrm{e}, \mathrm{q}) \leq \mu_{\varphi}^{+}(\mathrm{x}, \mathrm{q})$ and $\quad \mu_{\varphi}^{-}(\mathrm{e}, \mathrm{q}) \geq \mu_{\varphi}^{-}(\mathrm{x}, \mathrm{q})$ for all $\mathrm{x} \varepsilon \mathrm{X}$. Proof: Let $\mathrm{x} \& \mathrm{X}$, then

$\mu_{\varphi}^{+}(\mathrm{e}, \mathrm{q})=\mu_{\varphi}^{+}\left(\mathrm{x} \mathrm{x}^{-1}, \mathrm{q}\right) \leq \mathrm{T}\left\{\mu_{\varphi}^{+}(\mathrm{x}, \mathrm{q}), \mu_{\varphi}^{+}\left(\mathrm{x}^{-1}, \mathrm{q}\right)\right\} \leq \mathrm{T} \quad\{$ $\left.\mu_{\varphi}^{+}(\mathrm{x}, \mathrm{q}), \mu_{\varphi}^{+}(\mathrm{x}, \mathrm{q})\right\}$

$\leq \mu_{\varphi}^{+}(\mathrm{x}, \mathrm{q})$

and $\mu_{\varphi}^{-}(\mathrm{e}, \mathrm{q})=\mu_{\varphi}^{-}\left(\mathrm{x} \mathrm{x}^{-1}, \mathrm{q}\right) \geq \mathrm{S}\left\{\mu_{\varphi}^{-}(\mathrm{x}, \mathrm{q}), \mu_{\varphi}^{-}\left(\mathrm{x}^{-1}, \mathrm{q}\right)\right\} \leq \mathrm{S}\left\{\mu_{\varphi}\right.$ $\left.(\mathrm{x}, \mathrm{q}), \mu_{\varphi}^{-}(\mathrm{x}, \mathrm{q})\right\}$

$\geq \mu_{\varphi}^{-}(\mathrm{x}, \mathrm{q})$

This completes the proof.

Proposition 3.2:Let ' $\phi$ ' be a bipolar anti Q- fuzzy group of $\mathrm{X}$, then the following assertations are valid.

(i) $(\forall \alpha \varepsilon[0,1]$

$\left(\phi_{\alpha}^{+} \neq \phi \Rightarrow \phi_{\mathrm{t}}^{+}\right.$is a group of $\left.\mathrm{X}\right)$

(ii) $(\forall \beta \varepsilon[-1,0]$

$\left(\phi_{\beta}{ }^{-} \neq \phi \Rightarrow\right.$

$\phi_{\beta}{ }^{-}$is a group of $\left.\mathrm{X}\right)$

Proof: Let $\mathrm{t} \varepsilon[0,1]$ be such that $\phi_{\mathrm{t}}^{+} \neq \phi$. If $\mathrm{x}, \mathrm{y} \varepsilon \phi_{\mathrm{t}}^{+}$, then $\mu_{\phi}^{+}(\mathrm{x}, \mathrm{q}) \leq \mathrm{t}$ and $\quad \mu_{\phi}^{+}(\mathrm{y}, \mathrm{q}) \leq \mathrm{t}$. It follows that $\mu_{\phi}^{+}(\mathrm{xy}, \mathrm{q}) \leq \mathrm{T}\left\{\mu_{\phi}^{+}(\mathrm{x}, \mathrm{q}), \mu_{\phi}^{+}(\mathrm{y}, \mathrm{q})\right\} \leq \mathrm{t}$

Corollary 3.3: If $\phi$ is a bipolar anti Q- fuzzy group of $\mathrm{X}$, then the sets $\phi_{\mu \varphi+(e, q)}^{+}$and $\quad \phi_{\mu \varphi}^{-}(\mathrm{e}, \mathrm{q})$ are group of $\mathrm{X}$.

Proof: Straight forward.

Proposition 3.4: Let $\phi=\left(\mathrm{X}, \mu_{\phi}^{+}, \mu_{\phi}^{-}\right)$be a bipolar anti Qfuzzy d-ideal of $X$. If the inequality $x y \geq z$ holds in $X$, then

$$
\begin{aligned}
& \mu_{\phi}^{+}(\mathrm{x}, \mathrm{q}) \leq \mathrm{T}\left\{\mu_{\phi}^{+}(\mathrm{y}, \mathrm{q}), \mu_{\phi}^{+}(\mathrm{z}, \mathrm{q})\right\} \\
& \mu_{\phi}^{-}(\mathrm{x}, \mathrm{q}) \geq \mathrm{S}\left\{\mu_{\phi}^{-}(\mathrm{y}, \mathrm{q}), \mu_{\phi}^{-}(\mathrm{z}, \mathrm{q})\right\}
\end{aligned}
$$

Proof: $\quad$ Let $x, y, z \in X$ be such that $x y \geq z$, then $(\mathrm{xy}) \mathrm{z}=0$, and so

$$
\begin{aligned}
\mu_{\phi}^{+}(\mathrm{x}, \mathrm{q}) \leq \mathrm{T}\left\{\mu_{\phi}^{+}(\mathrm{xy}, \mathrm{q}), \mu_{\phi}^{+}(\mathrm{y}, \mathrm{q})\right\} \\
\leq \mathrm{T}\left\{\mathrm{T}\left\{\mu_{\phi}^{+}(\mathrm{xy}, \mathrm{q}) \mathrm{z}, \mu_{\phi}^{+}(\mathrm{z}, \mathrm{q})\right\},\right.
\end{aligned}
$$

$\left.\mu_{\phi}^{+}(\mathrm{y}, \mathrm{q})\right\}$

$$
=\mathrm{T}\left\{\mathrm{T}\left\{\mu_{\phi}^{+}(\mathrm{e}, \mathrm{q}), \mu_{\phi}^{+}(\mathrm{z}, \mathrm{q})\right\},\right.
$$

$\left.\mu_{\phi}^{+}(\mathrm{y}, \mathrm{q})\right\}$

$$
=\mathrm{T}\left\{\mu_{\phi}^{+}(\mathrm{y}, \mathrm{q}), \mu_{\phi}^{+}(\mathrm{z}, \mathrm{q})\right\}
$$

and 


$$
\geq \mathrm{S}\left\{\mathrm{S}\left\{\mu_{\phi}^{-}(\mathrm{xy}, \mathrm{q}) \mathrm{z}, \mu_{\phi}^{-}(\mathrm{z})\right\}, \mu_{\phi}^{-}\right.
$$

$(\mathrm{y}, \mathrm{q})\}$

$$
=\mathrm{S}\left\{\mathrm{S}\left\{\mu_{\phi}^{-}(\mathrm{e}, \mathrm{q}), \mu_{\phi}^{-}(\mathrm{z}, \mathrm{q})\right\}, \mu_{\phi}^{-}\right.
$$

$(\mathrm{y}, \mathrm{q})\}$

$$
=\mathrm{S}\left\{\mu_{\phi}^{-}(\mathrm{y}, \mathrm{q}), \mu_{\phi}^{-}(\mathrm{z}, \mathrm{q})\right\}
$$

This completes the proof.

Proposition 3.5: Let $\phi$ be a bipolar anti Q- fuzzy d-ideal of X. If the inequality $\mathrm{x} \geq \mathrm{y}$ holds in $\mathrm{X}$, then $\mu_{\phi}^{+}(\mathrm{x}, \mathrm{q}) \leq \mu_{\phi}^{+}(\mathrm{y}, \mathrm{q})$ and $\mu_{\phi}^{-}(\mathrm{x}, \mathrm{q}) \geq \mu_{\phi}^{-}(\mathrm{y}, \mathrm{q})$.

Proof: Let $\mathrm{x}, \mathrm{y} \varepsilon \mathrm{X}$ be such that $\mathrm{x} \geq \mathrm{y}$, then $\mu_{\phi}^{+}(\mathrm{x}, \mathrm{q}) \leq \mathrm{T}\left\{\mu_{\phi}^{+}(\mathrm{xy}, \mathrm{q}), \mu_{\phi}^{+}(\mathrm{y}, \mathrm{q})\right\}=\mathrm{T}\left\{\mu_{\phi}^{+}(\mathrm{e}, \mathrm{q})\right.$, $\left.\mu_{\phi}^{+}(\mathrm{y}, \mathrm{q})\right\}=\mu_{\phi}^{+}(\mathrm{y}, \mathrm{q})$ $\mu_{\phi}^{-}(\mathrm{x}, \mathrm{q}) \geq \mathrm{S}\left\{\mu_{\phi}^{-}\right.$

$\left.(\mathrm{xy}, \mathrm{q}), \mu_{\phi}^{-}(\mathrm{y}, \mathrm{q})\right\}=\mathrm{T}\left\{\mu_{\phi}^{-}(\mathrm{e}, \mathrm{q}), \mu_{\phi}^{-}(\mathrm{y}, \mathrm{q})\right\} \quad=\mu_{\phi}^{-}(\mathrm{y}, \mathrm{q})$

This completes the proof.

Proposition 3.6: In a group $X$, every bipolar anti Q- fuzzy dideal of $\mathrm{X}$ is bipolar anti Q- fuzzy group of $\mathrm{X}$.

Proof: Let ' $\phi$ ' be a bipolar anti Q- fuzzy d-ideal of a group $X$. Since $x y \geq x$ for all $x, y \in X$, it follows from Proposition (3.5) that

$\mu_{\phi}^{+}(\mathrm{xy}, \mathrm{q}) \leq \mathrm{T}\left\{\mu_{\phi}^{+}(\mathrm{x}, \mathrm{q}), \mu_{\phi}^{+}(\mathrm{y} \mathrm{q}),\right\}$ and $\mu_{\phi}^{-}(\mathrm{x}, \mathrm{q}) \geq \mu_{\phi}^{-}(\mathrm{x}, \mathrm{q})$, so from Proposition 3.1

$\left(\mathrm{BPFG}_{1}\right) \mu_{\phi}^{+}(\mathrm{xy}, \mathrm{q}) \leq \mathrm{T}\left\{\mu_{\phi}^{+}(\mathrm{x}, \mathrm{q}) \leq \mathrm{T}\left\{\mu_{\phi}^{+}(\mathrm{xy}, \mathrm{q}), \mu_{\phi}^{+}(\mathrm{y}, \mathrm{q})\right\}=\right.$ $\mathrm{T}\left\{\mu_{\phi}^{+}(\mathrm{x}, \mathrm{q}), \mu_{\phi}^{+}(\mathrm{y}, \mathrm{q})\right\}$

and $\quad \mu_{\phi}^{-}(\mathrm{xy}, \mathrm{q}) \geq \mu_{\phi}^{-}(\mathrm{x}, \mathrm{q}) \geq \mathrm{S}\left\{\mu_{\phi}^{-}(\mathrm{xy}, \mathrm{q}), \mu_{\phi}^{-}(\mathrm{y}, \mathrm{q})\right\} \geq \mathrm{S}\{$ $\left.\mu_{\phi}^{-}(\mathrm{x}, \mathrm{q}), \mu_{\phi}^{-}(\mathrm{y}, \mathrm{q})\right\}$

$$
\mu_{\phi}^{+}\left(x^{-1}, q\right) \leq \mathrm{T}\left\{\mu_{\phi}^{+}(\mathrm{xy}, \mathrm{q}), \mu_{\phi}^{+}(\mathrm{x}, \mathrm{q})\right\}=\mathrm{T}\{
$$$$
\left.\mu_{\phi}^{+}(\mathrm{e}, \mathrm{q}), \mu_{\phi}^{+}(\mathrm{y}, \mathrm{q})\right\} \leq \mu_{\phi}^{+}(\mathrm{x}, \mathrm{q})
$$$$
\mu_{\phi}^{-}\left(x^{-1}, q\right) \geq S\left\{\mu_{\phi}^{-}(x y, q), \mu_{\phi}^{-}(y, q)\right\}
$$

$$
\geq \mathrm{S}\left\{\mu_{\phi}^{-}(\mathrm{e}, \mathrm{q}), \mu_{\phi}^{-}\right.
$$

$(\mathrm{y}, \mathrm{q})\} \geq \mu_{\phi}^{-}(\mathrm{x}, \mathrm{q})$

Hence $\phi$ is bipolar anti Q- fuzzy group. The converse of the theorem is not true in general.

Proposition 3.7: Let ' $\phi$ ' be a bipolar anti Q- fuzzy group of a group X such that Proposition 3.2 holds for all $\mathrm{x}, \mathrm{y}, \mathrm{z} \varepsilon \mathrm{X}$ satisfying the inequality $\mathrm{xy} \varepsilon \mathrm{z}$ then $\phi$ is a bipolar anti Qfuzzy d-ideal of $X$.

Proof:

Recall from Proposition 3.1 that $\mu_{\varphi}^{+}(\mathrm{e}, \mathrm{q}) \leq$ $\mu_{\varphi}^{+}(\mathrm{x}, \mathrm{q})$ and $\mu_{\varphi}{ }^{-}(\mathrm{e}, \mathrm{q}) \geq \mu_{\varphi}{ }^{-}(\mathrm{x}, \mathrm{q})$ for all $\mathrm{x} \varepsilon \mathrm{X}$. Since $\mathrm{x}(\mathrm{xy}) \geq \mathrm{y}$ for all $\mathrm{x}, \mathrm{y} \varepsilon \mathrm{X}$, it follows that Proposition 3.2 ,

$$
\begin{aligned}
& \mu_{\phi}^{+}(\mathrm{x}, \mathrm{q}) \leq \mathrm{T}\left\{\mu_{\phi}^{+}(\mathrm{xy}, \mathrm{q}), \mu_{\phi}^{+}(\mathrm{y}, \mathrm{q})\right\} \text { and } \\
& \mu_{\phi}^{-}(\mathrm{x}, \mathrm{q}) \geq \mathrm{S}\left\{\mu_{\phi}^{-}(\mathrm{xy}, \mathrm{q}), \mu_{\phi}^{-}(\mathrm{y}, \mathrm{q})\right\} .
\end{aligned}
$$

Hence $\phi$ is a bipolar anti Q- fuzzy d-ideal of X.

Proposition 3.8: Let $\lambda$ and $\mu$ be bipolar anti Q- fuzzy d-ideal of $\mathrm{X}$, then $\lambda \times \mu$ is also bipolar anti Q- fuzzy d-ideal of $\mathrm{X}$.

Proof: $\quad$ For any $\left(\mathrm{x}_{1}, \mathrm{x}_{2}\right),\left(\mathrm{y}_{1}, \mathrm{y}_{2}\right) \varepsilon \mathrm{X} \times \mathrm{X}$, we have $\left(\mathrm{BFd}_{1}\right) \quad\left(\lambda^{+} \times \mu^{+}\right)_{\mathrm{q}}\left(\mathrm{x}_{1}, \mathrm{x}_{2}\right)=\mathrm{T}\left\{\lambda^{+}\left(\mathrm{x}_{1}, \mathrm{q}\right), \mu^{+}\left(\mathrm{x}_{2}, \mathrm{q}\right)\right\}$ $\leq \mathrm{T}\left\{\mathrm{T}\left\{\lambda^{+}\left(\mathrm{x}_{1}, \mathrm{y}_{1}\right)\right.\right.$,

$\left.\left.\lambda^{+}\left(\mathrm{y}_{1}, \mathrm{q}\right)\right\}, \mathrm{T}\left\{\mu^{+}\left(\mathrm{x}_{2}, \mathrm{y}_{2}\right), \mu^{+}\left(\mathrm{y}_{2}, \mathrm{q}\right)\right\}\right\}$

$=\mathrm{T}\left\{\mathrm{T}\left\{\lambda^{+}\left(\mathrm{x}_{1}, \mathrm{y}_{1}\right)\right.\right.$, $\left.\mu^{+}\left(\mathrm{x}_{2}, \mathrm{y}_{2}\right)\right\}, \mathrm{T}\left\{\lambda^{+}\left(\mathrm{y}_{1}, \mathrm{q}\right), \mu^{+}\left(\mathrm{y}_{2}, \mathrm{q}\right)\right\}$

$$
\left(\lambda^{-} \times \mu^{-}\right)_{\mathrm{q}}\left(\mathrm{x}_{1}, \mathrm{x}_{2}\right) \quad=\mathrm{S}\left\{\lambda^{+}\left(\mathrm{x}_{1}, \mathrm{q}\right), \mu^{+}\left(\mathrm{x}_{2}, \mathrm{q}\right)\right\}
$$

$\geq \mathrm{S}\left\{\mathrm{T}\left\{\lambda^{+}\left(\mathrm{x}_{1}, \mathrm{y}_{1}\right), \lambda^{+}\left(\mathrm{y}_{1}, \mathrm{q}\right)\right\}\right.$,

$\left.\mathrm{T}\left\{\mu^{+}\left(\mathrm{x}_{2}, \mathrm{y}_{2}\right), \mu^{+}\left(\mathrm{y}_{2}, \mathrm{q}\right)\right\}\right\}$

$=\mathrm{S}\left\{\mathrm{T}\left\{\lambda^{+}\left(\mathrm{x}_{1}, \mathrm{y}_{1}\right), \mu^{+}\left(\mathrm{x}_{2}\right.\right.\right.$, $\left.\left.\left.\mathrm{y}_{2}\right)\right\}, \mathrm{T}\left\{\lambda^{+}\left(\mathrm{y}_{1}, \mathrm{q}\right), \mu^{+}\left(\mathrm{y}_{2}, \mathrm{q}\right)\right\}\right\}$

Hence $\mu^{\mathrm{f}}$ is bipolar anti fuzzy d-ideal of $\mathrm{X}$.

Proposition 3.9: Let $\mathrm{f}: \mathrm{X} \rightarrow \mathrm{Y}$ be a homomorphism of groups. If ' $\mu$ ' is a bipolar anti

Q- fuzzy d-ideal of $y$, then $\mu^{\mathrm{f}}$ is bipolar anti Q-fuzzy d-ideal of X.

Proof:

For any $\mathrm{x} \varepsilon \mathrm{X}$, we have

$$
\begin{aligned}
& \mu^{+f}(\mathrm{x}, \mathrm{q})=\mu^{+}(\mathrm{f}(\mathrm{x}, \mathrm{q})) \leq \mu^{+}(\mathrm{e}, \mathrm{q})=\mu^{+}(\mathrm{f}(\mathrm{e}, \mathrm{q}))=\mu^{+\mathrm{f}}(\mathrm{e}, \mathrm{q}) \\
& \mu^{-\mathrm{f}}(\mathrm{x}, \mathrm{q})=\mu^{-}(\mathrm{f}(\mathrm{x}, \mathrm{q})) \geq \mu^{-}(\mathrm{e}, \mathrm{q})=\mu^{-}(\mathrm{f}(\mathrm{e}, \mathrm{q}))=\mu^{-\mathrm{f}}(\mathrm{e}, \mathrm{q})
\end{aligned}
$$

Let $\mathrm{x}, \mathrm{y} \& \mathrm{X}$

$$
\begin{aligned}
\mathrm{T}\left\{\mu^{+\mathrm{f}}(\mathrm{xy}, \mathrm{q}), \mu^{+\mathrm{f}}(\mathrm{y}, \mathrm{q})\right\}=\mathrm{T} & \left\{\mu ^ { + } \left(\mathrm{f}(\mathrm{xy}, \mathrm{q}), \mu^{+}(\mathrm{f}(\mathrm{y}, \mathrm{q})\}\right.\right. \\
& =\mathrm{T}\left\{\mu^{+}(\mathrm{f}(\mathrm{x}, \mathrm{q}) \cdot \mathrm{f}(\mathrm{y}, \mathrm{q})), \mu^{+}(\mathrm{f}(\mathrm{y}, \mathrm{q})\}\right. \\
& \geq \mu^{+} \mathrm{f}(\mathrm{x}, \mathrm{q})=\mu^{+\mathrm{f}}(\mathrm{x}, \mathrm{q}) .
\end{aligned}
$$

Proposition 3.10: Let $\mathrm{f}: \mathrm{X} \rightarrow \mathrm{Y}$ be an epimorphism of groups. If $\mu^{\mathrm{f}}$ is bipolar anti

Q- fuzzy d-ideal of $X$, then $\mu$ is bipolar anti Q-fuzzy d-ideal of Y.

Proof: $\quad$ Let $\mathrm{y} \varepsilon \mathrm{Y}$, there exists $\mathrm{x} \varepsilon \mathrm{X}$ such that $\mathrm{f}(\mathrm{x})$ $=\mathrm{y}$, then

$$
\mu^{+}(y, q)=\mu^{+}(f(x, q))=\mu^{+f}(x, q) \geq \mu^{+f}(e, q)=\mu^{+}(f(e, q))
$$
$=\mu^{+}(\mathrm{e}, \mathrm{q})$

$$
\mu^{-}(\mathrm{y}, \mathrm{q})=\mu^{-}(\mathrm{f}(\mathrm{x}, \mathrm{q}))=\mu^{-\mathrm{f}}(\mathrm{x}, \mathrm{q}) \leq \mu^{-\mathrm{f}}(\mathrm{e}, \mathrm{q})=\mu^{-}(\mathrm{f}(\mathrm{e}, \mathrm{q}))=
$$

$\mu^{-}(\mathrm{e}, \mathrm{q})$

Let $\mathrm{x}, \mathrm{y} \varepsilon \mathrm{Y}$, then there exists $\mathrm{a}, \mathrm{b} \varepsilon \mathrm{X}$, such that $\mathrm{f}(\mathrm{a}, \mathrm{q})=\mathrm{x}$ and $f(b, q)=y$. It follows that

$$
\mu^{+}(\mathrm{x}, \mathrm{q})=\mu^{+}(\mathrm{f}(\mathrm{a}, \mathrm{q}))=\mu^{+\mathrm{f}}(\mathrm{a}, \mathrm{q}) \text { and } \mu^{-}(\mathrm{x}, \mathrm{q})=\mu^{-}
$$

$(f(a, q))=\mu^{-f}(a, q)$

$$
\begin{aligned}
& \leq \mathrm{T}\left\{\mu^{+\mathrm{f}}(\mathrm{ab}, \mathrm{q}), \mu^{+\mathrm{f}}(\mathrm{b}, \mathrm{q})\right\} \\
& =\mathrm{T}\left\{\mu ^ { + } \left(\mathrm{f}(\mathrm{ab}, \mathrm{q}), \mu^{+}(\mathrm{f}(\mathrm{b}, \mathrm{q})\}\right.\right. \\
& =\mathrm{T}\left\{\mu^{+}(\mathrm{f}(\mathrm{a}, \mathrm{q}) \cdot \mathrm{f}(\mathrm{b}, \mathrm{q})), \mu^{+}(\mathrm{f}(\mathrm{b}, \mathrm{q})\}\right. \\
& =\mathrm{T}\left\{\mu^{+}(\mathrm{xy}, \mathrm{q}), \mu^{+}(\mathrm{y}, \mathrm{q})\right\}
\end{aligned}
$$

Also

$$
\begin{aligned}
& \geq \mathrm{S}\left\{\mu^{-\mathrm{f}}(\mathrm{ab}, \mathrm{q}), \mu^{-\mathrm{f}}(\mathrm{b}, \mathrm{q})\right\} \\
& =\mathrm{S}\left\{\mu ^ { - } \left(\mathrm{f}(\mathrm{ab}, \mathrm{q}), \mu^{-}(\mathrm{f}(\mathrm{b}, \mathrm{q})\}\right.\right.
\end{aligned}
$$




$$
\begin{aligned}
& =\mathrm{S}\left\{\mu^{-}(\mathrm{f}(\mathrm{a}, \mathrm{q}) \cdot \mathrm{f}(\mathrm{b}, \mathrm{q})), \mu^{-}(\mathrm{f}(\mathrm{b}, \mathrm{q})\}\right. \\
& =\mathrm{S}\left\{\mu^{-}(\mathrm{xy}, \mathrm{q}), \mu^{-}(\mathrm{y}, \mathrm{q})\right\}
\end{aligned}
$$

Hence $\mu$ is a bipolar anti Q- fuzzy d-ideal of $y$.

$$
=\mathrm{T}\left\{\left(\lambda^{+} \times \mu^{+}\right)_{\mathrm{q}}\left(\left(\mathrm{x}_{1}, \mathrm{x}_{2}\right),\left(\mathrm{y}_{1}, \mathrm{y}_{2}\right)\right\}\right.
$$

$\left(\lambda^{-} \times \mu^{-}\right)_{\mathrm{q}}\left(\mathrm{x}_{1}, \mathrm{x}_{2}\right)=\mathrm{S}\left\{\lambda^{-}\left(\mathrm{x}_{1}, \mathrm{q}\right), \mu^{-}\left(\mathrm{x}_{2}, \mathrm{q}\right)\right\}$

$\geq \mathrm{S}\left\{\mathrm{S}\left\{\lambda^{-}\left(\mathrm{x}_{1}, \mathrm{y}_{1}\right), \lambda^{-}\left(\mathrm{y}_{1}, \mathrm{q}\right)\right\}, \mathrm{S}\left\{\mu^{-}\left(\mathrm{x}_{2}, \mathrm{y}_{2}\right), \mu^{-}\left(\mathrm{y}_{2}, \mathrm{q}\right)\right\}\right.$

$=\mathrm{S}\left\{\mathrm{S}\left\{\lambda^{-}\left(\mathrm{x}_{1}, \mathrm{y}_{1}\right), \mu^{-}\left(\mathrm{x}_{2}, \mathrm{y}_{2}\right)\right\}, \mathrm{S}\left\{\lambda^{-}\left(\mathrm{y}_{1}, \mathrm{q}\right), \mu^{-}\left(\mathrm{y}_{2}, \mathrm{q}\right)\right\}\right.$

$=\mathrm{S}\left\{\left(\lambda^{-} \times \mu^{-}\right)_{\mathrm{q}}\left(\left(\mathrm{x}_{1}, \mathrm{x}_{2}\right)\left(\mathrm{y}_{1}, \mathrm{y}_{2}\right)\right),\left(\lambda^{-} \times \mu^{-}\right)_{\mathrm{q}}\left(\mathrm{y}_{1}, \mathrm{y}_{2}\right)\right\}$

$\left(\lambda^{+} \times \mu^{+}\right)_{\mathrm{q}}\left(\mathrm{x}_{1}^{-1}, \mathrm{x}_{2}^{-1}\right)=\mathrm{T}\left\{\lambda^{+}\left(\mathrm{x}_{1}^{-1}, \mathrm{q}\right), \mu^{+}\left(\mathrm{x}_{2}^{-1}, \mathrm{q}\right)\right\}$

$\leq \mathrm{T}\left\{\mathrm{T}\left\{\lambda^{+}\left(\mathrm{x}_{1}, \mathrm{y}_{1}\right), \quad \lambda^{+}\left(\mathrm{y}_{1}, \mathrm{q}\right)\right\}, \quad \mathrm{T}\{\right.$

$\left.\mu^{+}\left(\mathrm{x}_{2}, \mathrm{y}_{2}\right), \mu^{+}\left(\mathrm{y}_{2}, \mathrm{q}\right)\right\}$

$=\mathrm{T}\left\{\mathrm{T}\left\{\lambda^{+}\left(\mathrm{x}_{1}, \mathrm{y}_{1}\right), \quad \mu^{+}\left(\mathrm{x}_{2}, \mathrm{y}_{2}\right)\right\}, \quad \mathrm{T}\{\right.$

$\left.\lambda^{+}\left(\mathrm{y}_{1}, \mathrm{q}\right), \mu^{+}\left(\mathrm{y}_{2}, \mathrm{q}\right)\right\}$

$=\mathrm{T}\left\{\left(\lambda^{+} \times \mu^{+}\right)_{\mathrm{q}}\left(\left(\mathrm{x}_{1}, \mathrm{x}_{2}\right) \quad\left(\mathrm{y}_{1}, \mathrm{y}_{2}\right)\right)\right.$,

$\left.\left(\lambda^{+} \times \mu^{+}\right)_{\mathrm{q}}\left(\mathrm{y}_{1}, \mathrm{y}_{2}\right)\right\}$

$\left(\lambda^{-} \times \mu^{-}\right)_{\mathrm{q}}\left(\mathrm{x}_{1}^{-1}, \mathrm{x}_{2}^{-1}\right)=\mathrm{S}\left\{\lambda^{-}\left(\mathrm{x}_{1}^{-1}, \mathrm{q}\right), \lambda^{-}\left(\mathrm{x}_{2}^{-1}, \mathrm{q}\right)\right\}$ $\geq \mathrm{S}\left\{\mathrm{S}\left\{\lambda^{-}\left(\mathrm{x}_{1}, \mathrm{y}_{1}\right), \lambda^{-}\left(\mathrm{y}_{1}, \mathrm{q}\right)\right\}, \mathrm{S}\left\{\mu^{-}\left(\mathrm{x}_{2}, \mathrm{y}_{2}\right), \mu\right.\right.$

$\left.\left(\mathrm{y}_{2}, \mathrm{q}\right)\right\}$

$$
=S\left\{S\left\{\lambda^{-}\left(x_{1}, y_{1}\right), \mu^{-}\left(x_{2}, y_{2}\right)\right\}, S\left\{\lambda^{-}\left(y_{1}, q\right), \mu^{-}\right.\right.
$$

$\left.\left(\mathrm{y}_{2}, \mathrm{q}\right)\right\}$

$$
\geq S\left\{\left(\lambda^{-} \times \mu^{-}\right)_{q}\left(\left(x_{1}, x_{2}\right),\left(y_{1}, y_{2}\right)\right),\left(\lambda^{-} \times \mu^{-}\right.\right.
$$

)$\left._{4}\left(y_{1}, y_{2}\right)\right\}$

Hence $\lambda \times \mu$ is bipolar anti Q- fuzzy d-ideal of $X$.

Proposition 3.11: Let ' $A$ ' be a bipolar anti Q-fuzzy set in a group $\mathrm{X}$ and $\mu_{\mathrm{A}}$ be the strongest bipolar anti Q- fuzzy relation on $\mathrm{X}$, then $\mathrm{A}$ is a bipolar anti Q- fuzzy d-ideal of $\mathrm{X}$ if and only if $\mu_{\mathrm{A}}$ is a bipolar anti Q-fuzzy d-ideal of $X \times X$.

Proof: Suppose that 'A' is a bipolar anti Q- fuzzy d-ideal of $\mathrm{X}$, then

$$
\mu_{\mathrm{A}}^{+}(\mathrm{e}, \mathrm{e})_{\mathrm{q}}=\mathrm{T}\left\{\mathrm{A}^{+}(\mathrm{e}, \mathrm{q}), \mathrm{A}^{+}(\mathrm{e}, \mathrm{q})\right\}
$$$$
\leq \mathrm{T}\left\{\mathrm{A}^{+}(\mathrm{x}, \mathrm{q}), \mathrm{A}^{+}(\mathrm{y}, \mathrm{q})\right\}=\mu_{\mathrm{A}}^{+}(\mathrm{x},
$$

$\mathrm{y})_{\mathrm{q}}$ for all $(\mathrm{x}, \mathrm{y}) \varepsilon \mathrm{X} \times \mathrm{X}$.

$$
\mu_{\mathrm{A}}^{-}(\mathrm{e}, \mathrm{e})_{\mathrm{q}}=\mathrm{S}\left\{\mathrm{A}^{-}(\mathrm{e}, \mathrm{q}), \mathrm{A}^{-}(\mathrm{e}, \mathrm{q})\right\}
$$$$
\geq \mathrm{S}\left\{\mathrm{A}^{-}(\mathrm{x}, \mathrm{q}), \mathrm{A}^{-}(\mathrm{y}, \mathrm{q})\right\}=\mu_{\mathrm{A}}^{-}(\mathrm{x},
$$

$\mathrm{y})_{\mathrm{q}}$ for all $(\mathrm{x}, \mathrm{y}) \varepsilon \mathrm{X} \times \mathrm{X}$.

For any $\mathrm{x}=\left(\mathrm{x}_{1}, \mathrm{x}_{2}\right)$ and $\mathrm{y}=\left(\mathrm{y}_{1}, \mathrm{y}_{2}\right) \varepsilon \mathrm{X} \times \mathrm{X}$.

$$
\begin{aligned}
\mu_{\mathrm{A}}^{+}(\mathrm{x}, \mathrm{q})=\mu_{\mathrm{A}}^{+}\left(\mathrm{x}_{1}, \mathrm{x}_{2}\right)_{\mathrm{q}} & =\mathrm{T}\left\{\mathrm{A}^{+}\left(\mathrm{x}_{1}, \mathrm{q}\right), \mathrm{A}^{+}\left(\mathrm{x}_{2}, \mathrm{q}\right)\right\} \\
& \leq \mathrm{T}\left\{\mathrm{T}\left\{\mathrm{A}^{+}\left(\mathrm{x}_{1}, \mathrm{y}_{1}\right), \mathrm{A}^{+}\left(\mathrm{y}_{1}, \mathrm{q}\right)\right\},\right.
\end{aligned}
$$

$\left.\mathrm{T}\left\{\mathrm{A}^{+}\left(\mathrm{x}_{2}, \mathrm{y}_{2}\right), \mathrm{A}^{+}\left(\mathrm{y}_{2}, \mathrm{q}\right)\right\}\right\}$

$$
=\mathrm{T}\left\{\mathrm{T}\left\{\mathrm{A}^{+}\left(\mathrm{x}_{1}, \mathrm{y}_{1}\right), \mathrm{A}^{+}\left(\mathrm{x}_{2}, \mathrm{y}_{2}\right)\right\},\right.
$$

$\left.\mathrm{T}\left\{\mathrm{A}^{+}\left(\mathrm{y}_{1}, \mathrm{q}\right), \mathrm{A}^{+}\left(\mathrm{y}_{2}, \mathrm{q}\right)\right\}\right\}$
$=\mathrm{T}\left\{\mu_{\mathrm{A}}^{+}\left(\left(\mathrm{x}_{1}, \mathrm{y}_{1}\right)\left(\mathrm{x}_{2}, \mathrm{y}_{2}\right) \mathrm{q}\right)\right.$, $\left.\mu_{\mathrm{A}}^{+}\left(\mathrm{y}_{1}, \mathrm{y}_{2}\right) \mathrm{q}\right\}$

$=\mathrm{T}\left\{\mu_{\mathrm{A}}^{+}\left((\mathrm{xy}, \mathrm{q}), \mu_{\mathrm{A}}^{+}(\mathrm{y}, \mathrm{q})\right\}\right.$

$\mu_{\mathrm{A}^{-}}(\mathrm{x}, \mathrm{q})=\mu_{\mathrm{A}}^{-}\left(\mathrm{x}_{1}, \mathrm{x}_{2}\right) \mathrm{q} \quad=\mathrm{S}\left\{\mathrm{A}^{-}\left(\mathrm{x}_{1}, \mathrm{q}\right), \mathrm{A}^{-}\left(\mathrm{x}_{2}, \mathrm{q}\right)\right\}$

$\geq \mathrm{S}\left\{\mathrm{S}\left\{\mathrm{A}^{-}\left(\mathrm{x}_{1}, \mathrm{y}_{1}\right), \mathrm{A}^{-}\left(\mathrm{y}_{1}, \mathrm{q}\right\}\right.\right.$

$\mathrm{S}\left\{\mathrm{A}^{-}\left(\mathrm{x}_{2}, \mathrm{y}_{2}\right), \mathrm{A}^{-}\left(\mathrm{y}_{2}, \mathrm{q}\right)\right\}$

$=\mathrm{S}\left\{\mathrm{S}\left\{\mathrm{A}^{-}\left(\mathrm{x}_{1}, \mathrm{y}_{1}\right), \mathrm{A}^{-}\left(\mathrm{x}_{2}, \mathrm{y}_{2}\right)\right\}\right.$, $\left.\mathrm{S}\left\{\mathrm{A}^{-}\left(\mathrm{y}_{1}, \mathrm{q}\right), \mathrm{A}^{-}\left(\mathrm{y}_{2}, \mathrm{q}\right)\right\}\right\}$

$=\mathrm{S}\left\{\mu_{\mathrm{A}}^{-}\left(\left(\mathrm{x}_{1}, \mathrm{y}_{1}\right)\left(\mathrm{x}_{2}, \mathrm{y}_{2}\right) \mathrm{q}\right) \mu_{\mathrm{A}}\right.$ $\left.\left(\mathrm{y}_{1}, \mathrm{y}_{2}\right) \mathrm{q}\right\}$

$=\mathrm{S}\left\{\mu_{\mathrm{A}}^{-}(\mathrm{xy}, \mathrm{q}), \mu_{\mathrm{A}}^{-}(\mathrm{y}, \mathrm{q})\right\}$

Hence $\mu_{\mathrm{A}}$ is a bipolar anti Q-fuzzy d-ideal of $\mathrm{X} \times \mathrm{X}$. Conversely, suppose that $\mu_{\mathrm{A}}$ is a bipolar anti Q- fuzzy d-ideal of $\mathrm{X} \times \mathrm{X}$. Then,

$\mathrm{T}\left\{\mathrm{A}^{+}(\mathrm{e}, \mathrm{q}), \mathrm{A}^{+}(\mathrm{e}, \mathrm{q})\right\} \quad=\mu_{\mathrm{A}}^{+}(\mathrm{e}, \mathrm{e})_{\mathrm{q}} \leq \mu_{\mathrm{A}}^{+}(\mathrm{x}, \mathrm{y})_{\mathrm{q}}=$ $\mathrm{T}\left\{\mathrm{A}^{+}(\mathrm{x}, \mathrm{q}), \mathrm{A}^{+}(\mathrm{y}, \mathrm{q})\right\} \forall(\mathrm{x}, \mathrm{y}) \varepsilon \mathrm{X} \times \mathrm{X}$.

$\mathrm{S}\left\{\mathrm{A}^{-}(\mathrm{e}, \mathrm{q}), \mathrm{A}^{-}(\mathrm{e}, \mathrm{q})\right\} \quad=\mu_{\mathrm{A}^{-}}(\mathrm{e}, \mathrm{e})_{\mathrm{q}} \leq \mu_{\mathrm{A}}^{-}(\mathrm{x}, \mathrm{y})_{\mathrm{q}}=\mathrm{S}\left\{\mathrm{A}^{-}\right.$ $\left.(\mathrm{x}, \mathrm{q}), \mathrm{A}^{-}(\mathrm{y}, \mathrm{q})\right\}$

for any $\mathrm{x}=\left(\mathrm{x}_{1}, \mathrm{y}_{1}\right)$ and $\mathrm{y}=\left(\mathrm{y}_{1}, \mathrm{y}_{2}\right) \varepsilon \mathrm{X} \times \mathrm{X}$., we have

$\mathrm{T}\left\{\mathrm{A}\left(\mathrm{x}_{1}, \mathrm{q}\right), \mathrm{A}\left(\mathrm{x}_{2}, \mathrm{q}\right)\right\} \quad=\mu_{\mathrm{A}}\left(\mathrm{x}_{1}, \mathrm{x}_{2}\right)_{\mathrm{q}}$

$\left.\left.\mathrm{y}_{2}\right)_{\mathrm{q}}\right\}$

$\leq \mathrm{T}\left\{\mu_{\mathrm{A}}\left(\left(\mathrm{x}_{1}, \mathrm{x}_{2}\right)_{\mathrm{q}},\left(\mathrm{y}_{1}, \mathrm{y}_{2}\right)\right), \mu_{\mathrm{A}}\left(\mathrm{y}_{1}\right.\right.$,

$\left.\left.\mathrm{y}_{2}\right)_{\mathrm{q}}\right\}$

$\mathrm{T}\left\{\mathrm{A}\left(\mathrm{y}_{1}, \mathrm{q}\right), \mathrm{A}\left(\mathrm{y}_{2}, \mathrm{q}\right)\right\}$

$\left.\mathrm{T}\left\{\mathrm{A}\left(\mathrm{x}_{2}, \mathrm{y}_{2}\right)_{\mathrm{q}}, \mathrm{A}\left(\mathrm{y}_{2}, \mathrm{q}\right)\right\}\right\}$

$=\mathrm{T}\left\{\mu_{\mathrm{A}}\left(\mathrm{x}_{1} \mathrm{y}_{1}, \quad \mathrm{x}_{2} \mathrm{y}_{2}\right)_{\mathrm{q}}\right), \quad \mu_{\mathrm{A}}\left(\mathrm{y}_{1}\right.$,

$=\mathrm{T}\left\{\mathrm{T}\left\{\mathrm{A}\left(\mathrm{x}_{1}, \mathrm{y}_{1}\right)_{\mathrm{q}}, \mathrm{A}\left(\mathrm{x}_{2}, \mathrm{y}_{2}\right)_{\mathrm{q}}\right\}\right.$

$=T\left\{T\left\{A\left(x_{1}, y_{1}\right)_{q}, A\left(y_{1}, q\right)\right.\right.$,

Putting $\mathrm{x}_{1}=\mathrm{x}_{2}=0$, we have

$\mu_{\mathrm{A}}\left(\mathrm{x}_{1}, \mathrm{q}\right) \leq \mathrm{T}\left\{\mu_{\mathrm{A}}\left(\mathrm{x}_{1}, \mathrm{y}_{1}\right)_{\mathrm{q}}, \mu_{\mathrm{A}}\left(\mathrm{y}_{1}, \mathrm{q}\right)\right\}$

Likewise, $\mu_{\mathrm{A}}\left(\mathrm{x}_{1} \mathrm{y}_{1) \mathrm{q}} \leq \mathrm{T}\left\{\mu_{\mathrm{A}}\left(\mathrm{x}_{1}, \mathrm{q}\right), \mu_{\mathrm{A}}\left(\mathrm{x}_{2}, \mathrm{q}\right)\right\}\right.$

$\mathrm{S}\left\{\mathrm{A}\left(\mathrm{x}_{1}, \mathrm{q}\right), \mathrm{A}\left(\mathrm{x}_{2}, \mathrm{q}\right)\right\}=\mu_{\mathrm{A}}\left(\mathrm{x}_{1}, \mathrm{x}_{2}\right)_{\mathrm{q}}$

$\leq \mathrm{S}\left\{\mu_{\mathrm{A}}\left(\left(\mathrm{x}_{1}, \mathrm{x}_{2}\right)_{\mathrm{q}},\left(\mathrm{y}_{1}, \mathrm{y}_{2}\right)\right), \mu_{\mathrm{A}}\left(\mathrm{y}_{1}\right.\right.$,

$\left.\left.\mathrm{y}_{2}\right)_{\mathrm{q}}\right\}$

$\left.\left.\mathrm{y}_{2}\right)_{\mathrm{q}}\right\}$

$\mathrm{S}\left\{\mathrm{A}\left(\mathrm{y}_{1}, \mathrm{q}\right), \mathrm{A}\left(\mathrm{y}_{2}, \mathrm{q}\right)\right\}$

$=\mathrm{S}\left\{\mu_{\mathrm{A}}\left(\left(\mathrm{x}_{1} \mathrm{y}_{1}, \mathrm{x}_{2} \mathrm{y}_{2}\right)_{\mathrm{q}}\right), \mu_{\mathrm{A}}\left(\mathrm{y}_{1}\right.\right.$

$=\mathrm{S}\left\{\mathrm{S}\left\{\mathrm{A}\left(\mathrm{x}_{1}, \mathrm{y}_{1}\right)_{\mathrm{q}}, \mathrm{A}\left(\mathrm{x}_{2}, \mathrm{y}_{2}\right)_{\mathrm{q}}\right\}\right.$,

$=S\left\{S\left\{A\left(x_{1}, y_{1}\right)_{q}, A\left(y_{1}, q\right)\right.\right.$

$\mathrm{S}\left\{\mathrm{A}\left(\mathrm{x}_{2}, \mathrm{y}_{2}\right)_{\mathrm{q}}, \mathrm{A}\left(\mathrm{y}_{2}, \mathrm{q}\right)\right\}$

Putting $\mathrm{x}_{1}=\mathrm{x}_{2}=0$, we have

,$\mu_{\mathrm{A}}\left(\mathrm{x}_{1}, \mathrm{q}\right) \geq \mathrm{S}\left\{\mu_{\mathrm{A}}\left(\mathrm{x}_{1}, \mathrm{y}_{1}\right)_{\mathrm{q}}, \mu_{\mathrm{A}}\left(\mathrm{y}_{1}, \mathrm{q}\right)\right\}$

Likewise, $\mu_{\mathrm{A}}\left(\mathrm{x}_{1} \mathrm{y}_{1}\right)_{\mathrm{q}} \geq \mathrm{S}\left\{\mu_{\mathrm{A}}\left(\mathrm{x}_{1}, \mathrm{q}\right), \mu_{\mathrm{A}}\left(\mathrm{x}_{2}, \mathrm{q}\right)\right\}$

Hence $\mathrm{A}$ is a bipolar anti Q- fuzzy d-ideal of $\mathrm{X}$. 
Proposition 3.12: Let $\phi$ be a bipolar anti Q- fuzzy set in X, then $\phi$ is a bipolar anti Q-fuzzy d-ideal of $\mathrm{X}$ if and only if it satisfies the following assertations.

$$
\begin{array}{ll}
(\forall \alpha \varepsilon[0,1] & \left(\phi_{\mathrm{t}}^{+} \neq \phi \Rightarrow \phi_{\mathrm{t}}^{+} \text {is an ideal of } \mathrm{X}\right) \\
(\forall \beta \varepsilon[-1,0] & \left(\phi_{\mathrm{s}}{ }^{-} \neq \phi \Rightarrow \phi_{\beta}{ }^{-} \text {is an ideal of } \mathrm{X}\right)
\end{array}
$$

Proof: Assume that $\phi$ is a bipolar anti fuzzy Q- d-ideal of $\mathrm{X}$. Let $(\mathrm{s}, \mathrm{t}) \varepsilon[-1,0] \varepsilon[0,1]$ be such that $\phi_{\mathrm{t}}{ }^{+} \neq \phi$ and $\phi_{\mathrm{s}}{ }^{-} \neq \phi$.

Obviously, e $\varepsilon \phi_{\mathrm{t}}^{+} \cap \phi_{\mathrm{s}}{ }^{-}$.

Let $\mathrm{x}, \mathrm{y} \varepsilon \mathrm{X}$ be such that $\mathrm{xy} \varepsilon \phi_{\mathrm{t}}^{+}$and $\mathrm{y} \varepsilon \phi_{\mathrm{t}}^{+}$, and

Let $\mathrm{a}, \mathrm{b} \varepsilon \mathrm{X}$ be such that $\mathrm{ab} \varepsilon \phi_{\mathrm{s}}{ }^{-}$and $\mathrm{b} \varepsilon \phi_{\mathrm{s}}{ }^{-}$, then

$\mu_{\phi}^{+}(x y, q) \leq t, \quad \mu_{\phi}^{+}(y, q) \leq t, \mu_{\phi}^{-}(a b, q) \geq s$ and $\mu_{\phi}{ }^{-}(b, q)$

$\geq \mathrm{s}$.

It follows from Proposition 3.1

$$
\begin{aligned}
& \mu_{\phi}^{+}(\mathrm{x}, \mathrm{q}) \leq \mathrm{T}\left\{\mu_{\phi}^{+}(\mathrm{xy}, \mathrm{q}), \mu_{\phi}^{+}(\mathrm{y}, \mathrm{q})\right\} \leq \mathrm{t} \quad \text { and } \\
& \mu_{\phi}^{-}(\mathrm{a}, \mathrm{q}) \geq \mathrm{S}\left\{\mu_{\phi}^{-}(\mathrm{ab}, \mathrm{q}), \mu_{\phi}^{-}(\mathrm{b}, \mathrm{q}) \geq \mathrm{s}\right.
\end{aligned}
$$

so that $\mathrm{x} \varepsilon \phi_{\mathrm{t}}{ }^{+}$and a $\varepsilon \phi_{\mathrm{s}}{ }^{-}$. Therefore $\phi_{\mathrm{t}}{ }^{+}$and $\phi_{\mathrm{s}}{ }^{-}$are ideals of $\mathrm{X}$.

Conversely, suppose that the condition (corollary) is valid. For any $x \varepsilon X$, let $\mu_{\varphi}^{+}(x, q)=t$ and $\mu_{\varphi}{ }^{-}(x, q)=s$, then $x \varepsilon$ $\phi_{\mathrm{t}}{ }^{+} \cap \phi_{\mathrm{s}}{ }^{-}$, and so $\phi_{\mathrm{t}}{ }^{+}$and $\phi_{\mathrm{s}}{ }^{-}$are non-empty. Since $\phi_{\mathrm{t}}{ }^{+}$and $\phi_{\mathrm{s}}{ }^{-}$ are ideal of $\mathrm{X}$, e $\varepsilon \phi_{\mathrm{t}}{ }^{+} \cap \phi_{\mathrm{s}}{ }^{-}$. Hence $\mu_{\phi}{ }^{+}(\mathrm{e}, \mathrm{q}) \leq \mathrm{t}=\mu_{\phi}{ }^{+}(\mathrm{x}, \mathrm{q})$ and $\mu_{\phi}^{-}(e, q) \geq s=\mu_{\phi}^{-}(x, q)$ for all $x \varepsilon X$.

If there exists $x^{1}, y^{1}, a^{1}, b^{1} \varepsilon X$ such that $\mu_{\phi}^{+}\left(x^{1}, q\right) \geq$ $\mathrm{T}\left\{\mu_{\phi}^{+}\left(\mathrm{x}^{1} \mathrm{y}^{1}, \mathrm{q}\right), \mu_{\phi}^{+}\left(\mathrm{y}^{1}, \mathrm{q}\right)\right\}$ and $\mu_{\phi}{ }^{-}\left(a^{1}, q\right) \leq S\left\{\mu_{\phi}^{-}\left(a^{1} b^{1}, q\right), \mu_{\phi}^{-}\left(b^{1}, q\right)\right\}$ then by taking

$$
\begin{aligned}
& \mathrm{t}_{0}=1 / 2\left\{\mu_{\phi}^{+}\left(\mathrm{x}^{1}, \mathrm{q}\right)+\mathrm{T}\left\{\mu_{\phi}^{+}\left(\mathrm{x}^{1} \mathrm{y}^{1}, \mathrm{q}\right), \mu_{\phi}^{+}\left(\mathrm{y}^{1}, \mathrm{q}\right)\right\}\right. \\
& \mathrm{S}_{0}=1 / 2\left\{\mu_{\phi}{ }^{-}\left(\mathrm{a}^{1}, \mathrm{q}\right)+\mathrm{S}\left\{\mu_{\phi}^{-}\left(\mathrm{a}^{1} \mathrm{~b}^{1}, \mathrm{q}\right), \mu_{\phi}^{-}\left(\mathrm{b}^{1}, \mathrm{q}\right)\right\}\right.
\end{aligned}
$$

We have,

$$
\begin{aligned}
& \mu_{\phi}^{+}\left(x^{1}, q\right) \geq t_{0} \geq T\left\{\mu_{\phi}{ }^{+}\left(x^{1} y^{1}, q\right), \mu_{\phi}^{+}\left(y^{1}, q\right)\right\} \\
& \mu_{\phi}^{-}\left(a^{1}, q\right) \geq s_{0} \geq S\left\{\mu_{\phi}{ }^{-}\left(a^{1} b^{1}, q\right), \mu_{\phi}{ }^{-}\left(b^{1}, q\right)\right\} \\
& \text { Hence } x^{1} \notin \phi_{t 0}{ }^{+}, x^{1}, y^{1} \varepsilon \phi_{t 0}{ }^{+}, y^{1} \varepsilon \phi_{t 0}{ }^{+}, a^{1} \notin \phi_{\mathrm{s} 0}{ }^{-} \text {and }
\end{aligned}
$$

$\mathrm{b}^{1} \varepsilon \phi_{\mathrm{s} 0}$ - This is a contradiction and thus $\phi$ is a bipolar anti Qfuzzy d-ideal of $X$.

\section{CONCLUSION}

K.J. Lee introduces the notion of bipolar fuzzy sub algebra's and bipolar fuzzy ideals of BCK/BCI-algebra. In this paper, we provide a condition for a bipolar anti Q- fuzzy group and bipolar anti Q- fuzzy d-ideal. We give relations between a bipolar anti Q-fuzzy group and bipolar anti Qfuzzy d-ideal. We consider the concept of strongest bipolar anti Q- fuzzy relation and discuss some related properties.

\section{REFERENCES}

[1] M. Akram and K.H. Dar, 'On fuzzy d-algebras', Punjab University Journal of mathematics, 37(2005), 61-76.

[2] Dubois and H. Prade, Fuzzy sets and Systems; theory and applications, Academic Press, 1980.

[3] Y.B. Jun and S.Z. Song, Sub algebra's and Closed ideals of Belt-algebras based on bipolar-valued fuzzy sets, Sci. Math. Japan (submitted)

[4] K.M. Lee, Bipolar-Valued fuzzy sets and their operations, Proc. Int. Conf. on. Intelligent Technologies, Bangkok, Thailand (2000), 307-312.

[5] K.M. Lee, Comparison of interval valued fuzzy sets, Intuitionistic fuzzy sets and bipolar fuzzy sets, J. Fuzzy Logic Intelligent Systems, 14(2004), No.2, 125-129.

[6] K.J. Lee, Bipolar fuzzy sub algebra's, and bipolar fuzzy ideals of BCK/BCI-algebra's, Bulletin of Malaysian Mathematical Sciences Society, 1-14 (2008).

[7] A.Solairaju and R.Nagarajan, A New structure and Constructions of Q- fuzzy groups, Advances in Fuzzy Mathematics, 4(1)(2009).23-29.

[8] R.Nagarajan and A.Solairaju, Characterization of anti Qfuzzy R-sub modules over commutative rings, International Journal of Computer Applications, 5(3) (2010), 3-7.

[9] H.J. Zimmermann. Fuzzy set theory and its applications, Kluwer-Nijhoff Publishing, 1985. 